\title{
Antenna Beamforming for Energy Harvesting in Cognitive Radio Networks
}

\author{
Huidong Liu ${ }^{\star}$, Jin Chen ${ }^{\star}$, Guoru Ding ${ }^{\star \dagger}$, Theodoros A. Tsiftsis ${ }^{\ddagger}$, and Corbett Rowell ${ }^{\ddagger *}$ \\ ${ }^{\star}$ College of Communications Engineering, PLA University of Science and Technology, China \\ ${ }^{\dagger}$ National Mobile Communications Research Laboratory, Southeast University, Nanjing, China \\ ${ }^{\ddagger}$ Department of Electrical and Electronic Engineering, School of Engineering Nazarbayev University, Kazakhstan \\ * Department of Electronic and Computer Engineering, School of Engineering Hong Kong University of Science \& Technology, China \\ Email: brunojames@163.com, chenjin99@126.net, dingguoru@gmail.com, theodoros.tsiftsis@nu.edu.kz, corbett.rowell@nu.edu.kz
}

\begin{abstract}
In this paper, a cooperative cognitive radio network (CRN) with energy harvesting capabilities of its secondary users is considered. Specifically, cooperative spectrum sensing and multi-antenna beamforming are employed to improve the sensing performance and the energy transfer efficiency, respectively. In our approach, a homogeneous CRN scenario is studied where the optimal sensing probability of each second user (SU) is obtained to maximize the control center (CC) throughput while satisfying the energy causality and primary user (PU) collision constraints. An iterative algorithm is proposed to obtain the optimal charging time. Numerical results depict that in an energy constrained scenario, cooperative spectrum sensing with beamforming performs much better than cooperative spectrum sensing without beamforming in terms of increased system throughput.

Index Terms-Cooperative spectrum sensing, energy harvesting, energy cooperation, cognitive radio networks, energy beamforming
\end{abstract}

\section{INTRODUCTION}

Green mobile networks have received substantial attention from both academia and industry as a promising approach to increasing energy efficiency in response to the growing concerns about operational expenditures. Green networks address the global environmental cost of using fossil energy to power the cellular infrastructure [1]. Representative works include energy harvesting hardware and devices, energy-efficient communication techniques, energy-aware network architecture/protocol design, energy-friendly software applications, and renewable energy sources. Interest with regard to the powering mobile networks with renewable energy sources has increased substantially.

Since radio frequency (RF) signals that can carry energy and be used as a vehicle for transporting information at the same time, simultaneous wireless information and power transfer (SWIPT) proposed in [2] becomes an interesting new area of research. The authors in [3] studied the performance limits for multiple-input-multiple-output (MIMO) SWIPT systems and characterized achievable rate-energy tradeoffs for various practical receiver designs. In order to maximize the harvested energy, it is necessary to coordinate the transmit direction to the receiver (energy beamforming). The key for successful energy beamforming is the channel state information (CSI) knowledge at the transmitter side [4].

In addition to the CSI determination, another key design objective in wireless communication is the spectral efficiency which addresses the increasing spectrum demands of multimedia services. Cognitive radio networks (CRNs) are seen as promising technologies to achieve greater spectral efficiency [5]. Large portions of the licensed spectrum are seriously underutilized, leading the concept of opportunistic spectrum access, allowing secondary users to exploit underutilized spectrum gains [6].

Spectrum sensing is a well-recognized enabling technique for CRNs. Due to the hidden terminal problem, a secondary user (SU) may not notice the existence of the primary user (PU) and increase the interference to the licensed systems. One method to solve the above hidden terminal problem is allowing multiple cognitive users to cooperatively perform spectrum sensing. It has been shown that the performance of spectrum sensing can be improved with an increase of the number of cooperative partners [7] and can therefore overcome both the hidden terminal problem and poor channel conditions (multipath fading and shadowing) [8].

Recently, there has been research on CR systems using energy-harvesting techniques [9]. Optimal couple of sensing duration and detection threshold was studied in [10]. In our previous work, an RF-energy harvesting CRN employed cooperative spectrum sensing and an optimal cooperative spectrum sensing strategy was proposed to maximize the control center (CC) spectral efficiency [11]. Due to the limited antennas at the power source, however, the energy efficiency based on the aforementioned systems was difficult to satisfy the practical requirement without energy beamforming, especially when for long transfer distances.

In this paper, we consider beamforming RF-energy harvesting CR systems which use cooperative spectrum sensing in order to improve the sensing accuracy and the energy efficiency. The main contributions of this paper are twofold: i) A new mutually beneficial relationship between the $\mathrm{CC}$ and the $\mathrm{SU}$ is proposed and ii) An optimization problem to achieve the optimal sensing-throughout trade-off under the 
energy causality constraints is formulated and resolved.

In Section II, we describe the system model and formulate the expressions for the normalized effective throughput of the $\mathrm{CC}$. The proposed beam and sensing policy with optimal solution is presented in Section III together with our proposed iterative algorithm. We provide the numerical results and discussions in Section IV and conclude with Section V.

\section{SySTEM MODEL}

We consider a CRN where there are $N$ single-antenna SUs, one CC with $M$ antennas and one PU. Each SU has harvested energy to power its uplink information transmission and spectrum sensing. We assume that the $\mathrm{CC}$ and all SUs are perfectly synchronized and operate with a timedivision-duplexing (TDD) protocol. We consider frame-based transmissions over flat-fading channels on a single frequency band. The system consists of a licensed channel occupied by a PU transmitter. In each time slot, the PU occupies the spectrum with a probability $p_{0}$ and the spectrum is idle with probability $p_{i}=1-p_{0}$. As shown in Fig. $1, N$ SUs access the PU spectrum opportunistically. SUs use energy-harvesting techniques to recharge their batteries. Each SU employs a hard decision fusion scheme due to its higher energy and bandwidth efficiency over a soft fusion scheme along with a reliable detection performance that is asymptotically similar to that of a soft fusion scheme [12].

Fig. 1. Cooperative spectrum sensing exploiting RF-energy harvesting with multi-antenna beamforming

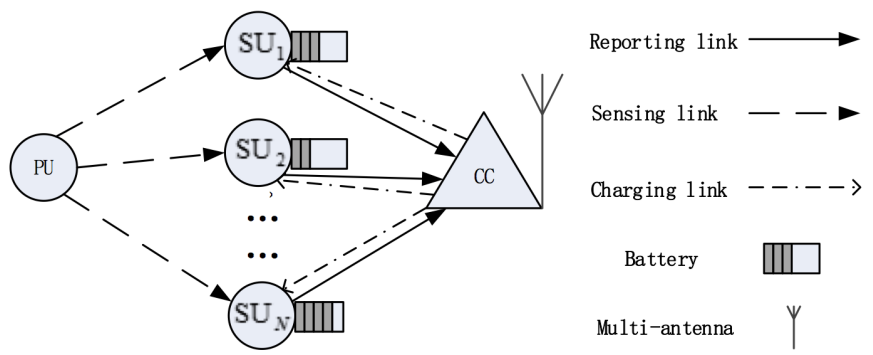

Fig. 2. Time Slot Structure

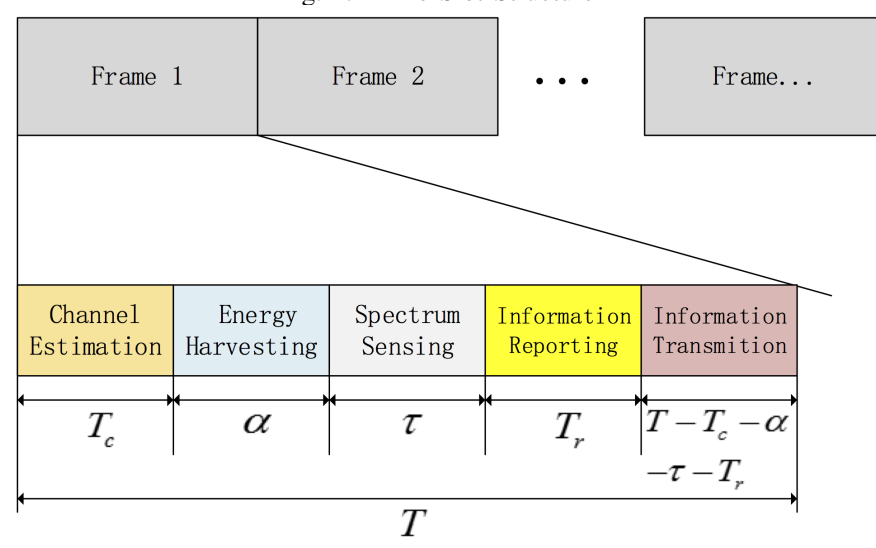

The time structure is shown in Fig. 2 and detailed as the following five steps:

- Channel estimation: during interval $\left(0, T_{c}\right]$, the SUs send orthogonal training pilots, and the $\mathrm{CC}$ estimates the channels and obtains the CSI by exploiting channel reciprocity.

- Energy harvesting: during interval $\left(T_{c}, \alpha\right]$, the $\mathrm{CC}$ transfers wireless energy to all SUs via energy beamforming with appropriately designed weights.

- Channel sensing: during interval $\left(T_{c}+\alpha, T_{c}+\alpha+\tau\right]$, the energy harvester stops working and the sensing receiver is powered on for sensing channel.

- Information reporting: during interval $\left(T_{c}+\alpha+\tau, T_{c}+\alpha+\right.$ $\tau+T_{r}$ ], every SU makes its own decision on the presence of the PU and then transmits its one bit decision to the CC.

- Data transmission: during interval $\left(T_{c}+\alpha+\tau+T_{r}, T\right]$, the $\mathrm{CC}$ accesses the channel to transmit information only when it senses that the PU is inactive meanwhile ensuring the specified PU protection level. For cooperative spectrum sensing, the CC makes a final decision on the PU's activity based on the received decisions.

Without loss of generality, we consider that all the SUs have the same sensing performance with the probability of detection $P_{d}$ and the probability of false alarm $P_{f}$. For the case of a complex valued phase shift keying PU signal with average received signal-to-noise ratio (SNR) $\gamma$ at each SU and circularly symmetric complex Gaussian noise with power $\sigma_{n}^{2}$, the $P_{d}$ and $P_{f}$ are given by [13]

$$
\begin{gathered}
P_{d}=Q\left(\left(\frac{\varepsilon}{\sigma_{n}^{2}}-\gamma-1\right) \sqrt{\frac{\tau f_{s}}{2 \gamma+1}}\right), \\
P_{f}=Q\left(\left(\frac{\varepsilon}{\sigma_{n}^{2}}-1\right) \sqrt{\tau f_{s}}\right),
\end{gathered}
$$

where $\varepsilon$ is the detection threshold and $f_{s}$ is the sampling rate. $Q(\cdot)$ denotes the complementary cumulative distribution function of a zero mean, unit variance Gaussian distribution.

The average normalized throughput of the $\mathrm{CC}$ with a time structure shown in Fig. 2 is given by

$$
R=\left(1-\frac{T_{\mathrm{c}}+\alpha+\tau+T_{r}}{T}\right)\left(1-Q_{f}(\tau, \varepsilon)\right) .
$$

where $Q_{f}(\tau, \varepsilon)=1-\prod_{n=1}^{N}\left(1-P_{f}(\tau, \varepsilon)\right)$.

From the above formula, it can be seen that the charging time $\alpha$, the sensing time $\tau$ and the threshold $\varepsilon$ have a great impact on the throughput. In the following part, we will discuss the optimal charging time, the sensing time, the sensing threshold, and the beamforming vector, which maximizes the throughput.

\section{Optimal Spectrum Sensing Policy}

The use of beamforming RF-energy harvesting technology for the SUs charging, increasing the SU's mobility, but too much charging time reduces the transmission time of the 
timeslot, reducing the throughput. Therefore we need to find the optimal energy beamformer, charging time, the sensing time and the threshold, which can achieve the maximum throughput under the condition of meeting the energy constraint and spectrum constraints.

The simplified problem formulation of the normalized throughput tradeoff with cooperative sensing exploiting beamforming RF-energy harvesting is given as

$$
\begin{gathered}
\max _{\alpha} R(\alpha) \\
\text { s. t. } A \leq \alpha \leq B .
\end{gathered}
$$

where $A=\frac{T_{c} P_{n}^{c}+T_{r} P_{n}^{r}}{p_{d} \beta_{n} M / N}, B=\frac{\left(T-T_{c}-T_{r}\right) P_{n}^{s}+T_{c} P_{n}^{c}+T_{r} P_{n}^{r}}{p_{d} \beta_{n} M / N+P_{n}^{s}}$

We then propose an iterative algorithm to find the solution of the $\alpha$ optimization problem (4-5). There is no closed-form solution for $\alpha$, hence, a search for the optimal $\alpha$ over $A \leq$ $\alpha \leq B$ is required. If $R(\alpha)$ is a unimodal function in the range of $A \leq \alpha \leq B$, however, instead of an exhaustive search, efficient search algorithms can be used.

Denote $\alpha^{*}$ as the optimal sensing time; $R(\alpha)$ is a unimodal function if it is monotonically increasing in $A \leq \alpha<\alpha^{*}$ and monotonically decreasing in $\alpha^{*}<\alpha \leq B$. Therefore, $R(\alpha)$ is the only local maximum in the entire range of $A \leq \alpha \leq B$.

Proposition: If the three conditions listed below are satisfied, then $R(\alpha)$ must be a unimodal function in the entire range of $A \leq \alpha \leq B$.

1) $\nabla R(A)>0$.

2) $\nabla R(B)<0$.

3) There is only one unique $\alpha^{*}$ where $A \leq \alpha \leq B$ such that $\nabla R\left(\alpha^{*}\right)=0$. where $\nabla$ denotes the differentiation of the function with respect to its argument.

The first condition means that $R(\alpha)$ is an increasing function at the point $\alpha=A$ and the second condition means that the function $R(\alpha)$ is decreasing at the point $\alpha=B$. Hence, these two conditions imply that there must be at least a point in $A<\alpha<B$ that maximizes $R(\alpha)$. If the third condition is true, then together with the first condition imply that $R(\alpha)$ is strictly increasing in $A \leq \alpha<\alpha^{*}$. Combining the second and third conditions implies that $R(\alpha)$ is strictly decreasing in $\alpha^{*}<\alpha \leq B$. Hence, the three conditions imply that $R(\alpha)$ must be the only local maximum in the entire range of $A \leq \alpha \leq B$ and $R(\alpha)$ is a unimodal function in that region.

We can prove that $R(\alpha)$ does satisfy all the three conditions for it to be a unimodal function in $A \leq \alpha \leq B$. The proofs are not shown in this paper due to the space constraint. Here, we give the expression of $\nabla R(\alpha)$ as follows,

$\nabla R(\alpha)=-\frac{1}{T P_{n}^{s}}\left(\left(D-Q_{f}(\alpha)\right)+\left(T P_{n}^{s}-\alpha D-C\right)\left(1-\nabla Q_{f}(\alpha)\right)\right.$

where

$$
D=P_{d} \beta_{n}(M+N-1) / N+P_{n}^{s},
$$

and

$$
\begin{aligned}
& \nabla Q_{f}(\alpha)=\frac{N \sqrt{D f_{s}}}{\sqrt{8 \pi \alpha}}\left(1-P_{f}(\alpha)^{N-1}\right) \\
& \cdot \exp \left(-\frac{\left(\sqrt{\frac{p_{d} \sqrt{2 \gamma+1}}{D f_{s} \alpha}} Q^{-1}\left(1-\sqrt[N]{1-\overline{P_{d}}}\right)+\sqrt{D f_{s} \alpha} \gamma\right)^{2}}{2}\right)
\end{aligned}
$$

Since $R(\alpha)$ is a unimodal function, efficient search algorithms such as Bisection search, Fibonacci search, Golden section search, etc., can be used to find the optimal $\alpha$. An example of using Bisection search method to find the optimal $\alpha$ is given in Algorithm 1.

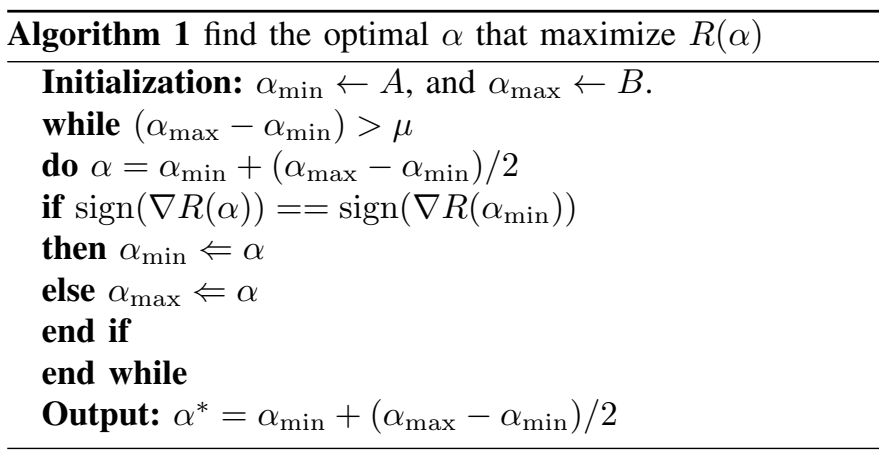

\section{PERformance Evaluation}

In this section, computer simulation results are presented to evaluate the throughput tradeoff with OR fusion rule where each SU is assumed to use the energy detector. Simulations are carried out to find $\alpha$ that simultaneously achieves the maximum $R$ and provides sufficient protection to the PU.

We set $M=50$, the number of SUs to be $N=3$, the frame duration, the estimating time and the reporting time are set to be $T=20 \mathrm{~ms}, T_{\mathrm{c}}=0 \mathrm{~ms}$ and $T_{r}=1 \mathrm{~ms}$, respectively. The bandwidth of the channel and the sampling frequency of the received signal are both assumed to be $6 \mathrm{MHz}$. We use the long-term fading model $\beta_{n}=d_{n}^{-3}$, where the distance $d_{n}=10$ $\mathrm{m}$ for all SUs. The estimating power, the sensing power, the reporting power and the transmit power of an SU are set as 2 $\mathrm{W}, 1 \mathrm{~W}, 2 \mathrm{~W}$ and $400 \mathrm{~W}$, respectively. The targeted probability of detection for the protection of the PU is set at $\overline{P_{d}}=95 \%$. Optimal values of was found when the SNR of the PU's signal received at the SUs is vary from $-30 \mathrm{~dB}$ to $0 \mathrm{~dB}$.

Fig. 3. Maximum normalized throughput with different $N$ SUs

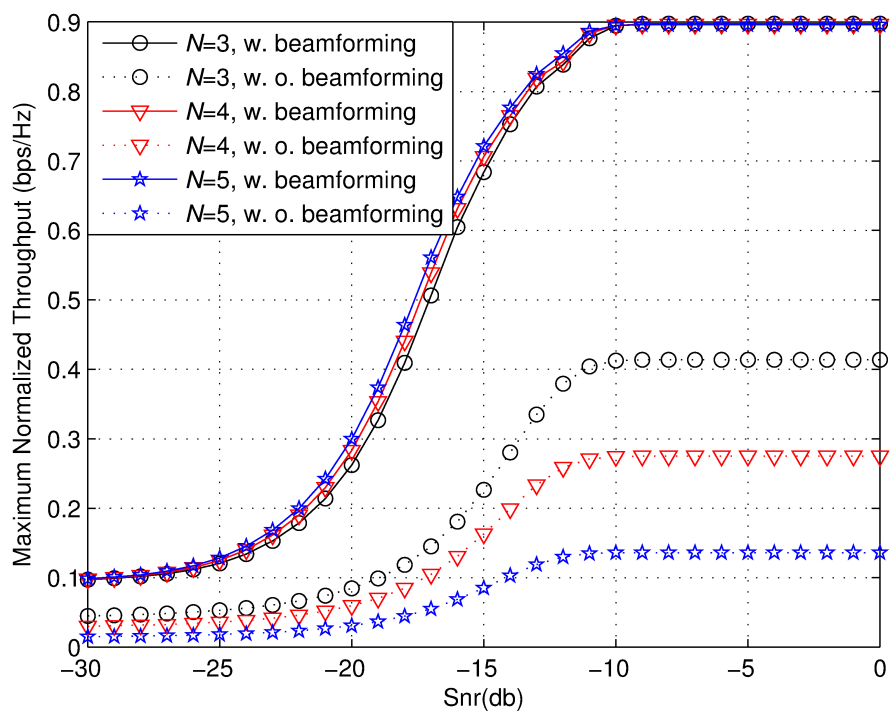


Fig. 3 compares the maximum normalized throughput when the number of the SUs is different. We can see that for $N=3$, 4 , 5, SUs, the maximum normalized throughput is larger with more cognitive radios in the system. Due to the increased SUs that can receive energy concurrently, the increased number of SUs is no longer a burden for the CC.

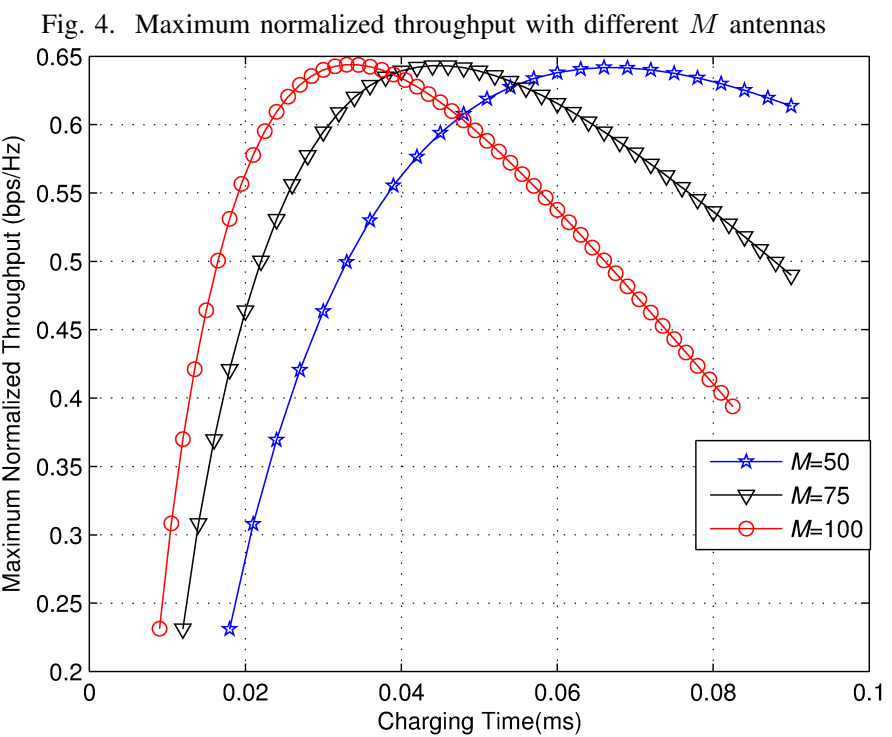

Fig. 4 shows the optimal throughput at different $M$ antennas. We can see that as $M$ increases, to reach the same energy, less time is needed to charge. Thus, we have more time to transmit and can get more throughput.

Fig. 5. Maximum normalized throughput with respects to transmit power

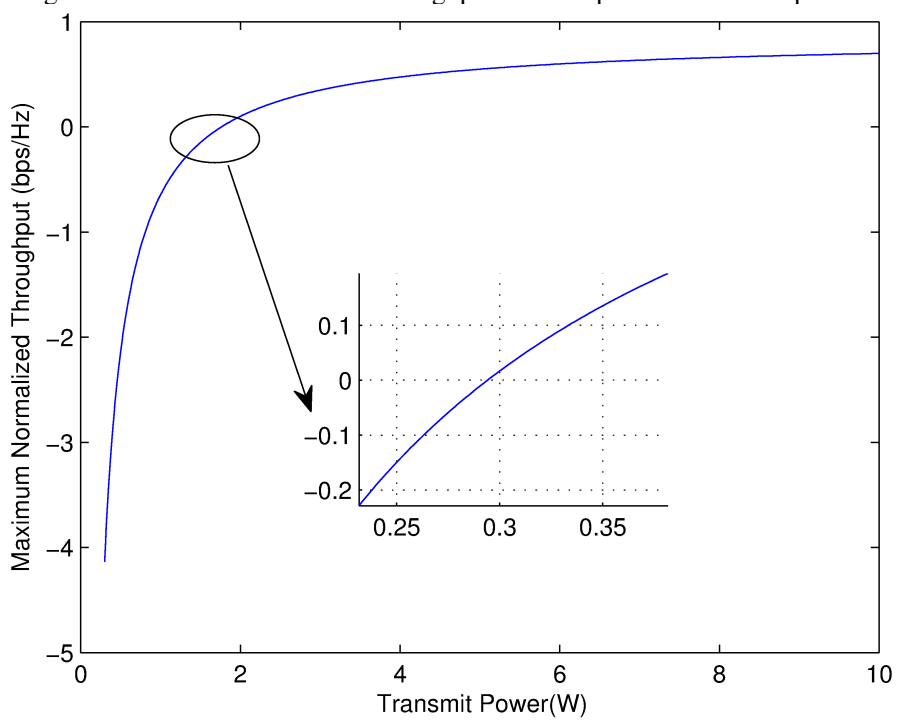

From Fig. 5, we can further see that when transmit power $p_{d}$ is less than $0.29 \mathrm{~W}$, the charging energy can not achieve the expected energy consumption and the system shuts down. Therefore, the lowest limit of transmit power $p_{d}$ need to be further analyzed in future works.

\section{CONCLUSION}

This paper proposed a new beamforming RF-energy harvesting CR system which employed cooperative spectrum sensing with OR fusion rule to improve the sensing accuracy and the transfer efficiency. An optimal sensing policy was derived to maximize the $\mathrm{CC}$ throughput while satisfying the PU collision and the energy causality constraints at each SU. Cooperative spectrum sensing with beamforming performs better than cooperative spectrum sensing without multiantenna beamforming in terms of increased system throughput. The sensing policy optimizes the CC throughput by selecting the appropriate charging time $\alpha$, sensing time $\tau$, and detection threshold $\varepsilon$. A greater value of transmit power $p_{d}$ can improve the CC throughput and low limit of transmit power $p_{d}$ need to be further analytically derived.

\section{ACKNOWLEDGEMENT}

This work is partially supported by the National Natural Science Foundation of China (Grant No. 61501510 and No. 61301160), and Natural Science Foundation of Jiangsu Province (Grant No. BK20150717).

\section{REFERENCES}

[1] C. Han, T. Harrold, S. Armour, I. Krikidis, S. Videv, P. Grant, H. Haas,J. Thompson, I. Ku, C.-X. Wang, T. A. Le, M. Nakhai, J. Zhang, and L. Hanzo, "Green radio: radio techniques to enable energy-efficient wireless networks," IEEE Commun. Mag., vol. 49, no. 6, pp. 46-54, June 2011

[2] R. Zhang and C. K. Ho, "MIMO broadcasting for simultaneous wireless information and power transfer," IEEE Trans. Wireless Commun., vol. 12, pp. 1989-2001, May 2013.

[3] X. Zhou, R. Zhang, and C. K. Ho, "Wireless information and power transfer: architecture design and rate-energy tradeoff," IEEE Trans. Commun., vol. 61, pp. 4757-4767, November 2013.

[4] S. Sanayei and A. Nosratinia, "Opportunistic beamforming with limited feedback,” IEEE Trans. Wireless Commun., vol. 6, no. 8, pp. 2765-2771, Aug. 2007.

[5] Q. Zhao and B. Sadler, "A survey of dynamic spectrum access," IEEE Signal Process. Mag., vol. 24, no. 3, pp. 79-89, May 2007.

[6] E. C. Y. Peh, Y.-C. Liang, and Y. L. Guan, " Optimization of cooperative sensing in cognitive radio networks: a sensing-throughput tradeoff view", in Proc. IEEE ICC, vol.58, pp.1-5, Sep. 2009.

[7] Ghasemi and E. S. Sousa, "Collaborative spectrum sensing for opportunistic access in fading environments," in Proc. IEEE DySPAN, pp. 131-136, Nov. 2005

[8] E. C. Y. Peh, Y.-C. Liang, Y. L. Guan, and Y. Pei, "Energy-efficient cooperative spectrum sensing in cognitive radio networks," in Proc. IEEE GLOBECOM, pp. 1-5, Dec. 2011.

[9] S. Park, H. Kim, and D. Hong, "Cognitive radio networks with energy harvesting," IEEE Trans. Wireless Commun., vol. 12, no. 3, pp. 13861397, Mar. 2013.

[10] W. Chung, S. Park, S. Lim, and D. Hong, "Spectrum sensing optimization for energy-harvesting cognitive radio systems," IEEE Trans. Wireless Commun., vol. 13, no. 5, pp. 2601-2613, May 2014.

[11] H. Liu, J. Chen, G. Ding, K. Yan, and L. Cui, "Optimal Cooperative Spectrum Sensing Strategy in Cognitive Radio Networks Exploiting RFEnergy Harvesting," in Proc. IEEE WCSP, Oct. 2015.

[12] S. M. Mishra, A. Sahai, and R. W. Brodensen, "Cooperative sensing among cognitive radios," in Proc. IEEE Int. Conf. Commun. ICC, pp. 1658-1663, June 2006.

[13] Y.-C. Liang, Y. Zeng, E. C. Y. Peh, and A. T. Hoang, "Sensing throughput tradeoff for cognitive radio networks," IEEE Trans. Wireless Commun., vol. 7, no. 4, pp. 1326-1337, Apr. 2008.

[14] G. Yang, C. K. Ho, R. Zhang, and Y. L. Guan, "Throughput optimization for massive MIMO systems powered by wireless energy transfer," IEEE J. Sel. Area. Commun., vol. 33, no. 8, pp. 1640-1650, Aug. 2015. 